\title{
Nonsuicidal self-harm in youth: a population-based survey
}

\author{
Mary K. Nixon MD, Paula Cloutier MA, S. Mikael Jansson PhD
}

\section{ABSTRACT}

Background: Nonsuicidal self-harm includes cutting, scratching, burning and minor overdosing. There have been few studies that have examined the rate of self-harm and mental-health correlates among community-based youth. We performed a population-based study to determine the prevalence of nonsuicidal self-harm, its mental-health correlates and help-seeking behaviour.

Methods: We used data from the Victoria Healthy Youth Survey, a population-based longitudinal survey of youth aged 14-21 in Victoria, British Columbia. The survey included questions about the history, method, frequency, age of onset and help-seeking for nonsuicidal self-harm. Youth were interviewed between February and June 2005. Univariable group differences were analyzed using students $t$ test for continuous data and $\chi^{2}$ for binary or categorical data. Multivariate analyses were conducted by use of multivariate analysis of variance and logistic regression.

Results: Ninety-six of 568 (16.9\%) youth indicated that they had ever harmed themselves. Self-injuries such as cutting, scratching and self-hitting were the most common forms of nonsuicidal self-harm ( $83.2 \%)$. The mean age of onset was 15.2 years. Of those who reported nonsuicidal self-harm, $56 \%$ had sought help for this behaviour. Participants who reported 5 or more symptoms (out of 6 ) in a given symptom category were more likely than those who reported less than 5 symptoms to report nonsuicidal selfharm for the following categories: depressive mood (odds ratio [OR] 2.18, confidence interval $[\mathrm{CI}] 1.28-3.7$ ) and problems with regulation of attention, impulsivity and activity (OR 2.24, $\mathrm{Cl} 1.33-3.76$ ).

Interpretation: We found a high lifetime prevalence of nonsuicidal self-harm. Many mental-health symptoms were associated with this behaviour, particularly those with depressive mood and attention-related problems. Just over half of youth reported seeking help for nonsuicidal selfharm. Clinicians who encounter youth should be vigilant to assess for this behaviour in youth who present with mental health issues.

Une version française de ce résumé est disponible à l'adresse www.cmaj.ca/cgi/content/full/I78/3/306/DCI

CMAJ 2008;178(3):306-12
$\mathrm{N}$ onsuicidal self-harm includes behaviours such as self-cutting, scratching and burning, done without the conscious intent to take one's life. Onset typically occurs between I4 and 24 years of age. ${ }^{1,2}$ The most common reasons for this type of harm are regulation of affect (e.g., to reduce tension or relieve dysphoric feelings), but reasons may also include self-punishment, interpersonal reasons, sensation seeking and anti-dissociation mechanisms. ${ }^{3}$ Factors associated with nonsuicidal self-harm include being female, awareness of self harm in peers, family members who self harm, drug misuse, depression, anxiety, impulsivity, disruptive disorders and low self-esteem. ${ }^{4,5}$ Suicide ideation and attempts are more likely to be reported among those with repeated nonsuicidal self-harm. ${ }^{6}$

A definition of "deliberate self-harm," which does not distinguish between suicidal and nonsuicidal intent, has been proposed by the Child and Adolescent Self-Harm in Europe research group and has been used in several schoolbased surveys of adolescents., ${ }^{4,7}$ The prevalence self-harm has been studied in school-based surveys of adolescents and young adults. ${ }^{4-6,8}$ The results of these surveys are of limited generalizability because the samples were restricted to schools in large urban centres. Thus, we performed a population-based survey of youth in western Canada to investigate nonsuicidal self-harm. Our objectives were to determine the rate of nonsuicidal self-harm among Canadian youth; to determine the mental-health factors associated with nonsuicidal self-harm; and to examine the help-seeking patterns for this behaviour.

\section{Methods}

\section{Study design and population}

We used cross-sectional data from the Victoria Healthy Youth Survey, a longitudinal study of the economic and psychological risks of unintentional injuries in youth. ${ }^{9}$ The data were collected in the Victoria, British Columbia, census metropolitan area (population 325 ooo). The first wave of the study was performed in 2003 and included youth aged $\mathrm{I} 2-\mathrm{I} 8$ years. In 2005, participants who agreed to participate in the first wave were re-surveyed (aged I4-2I years). Only the 2005 survey included questions about nonsuicidal self-harm.

From the Centre for Youth and Society (Nixon, Jansson), University of Victoria, Victoria, BC; and the Mental Health Patient Service Unit, Children's Hospital of Eastern Ontario (Cloutier), Ottawa, Ont. 
This study was approved by the human research ethics board at the University of Victoria, Victoria, British Columbia.

\section{Victoria Healthy Youth Survey}

Data were collected during a face-to-face interview in the youth's home or another agreed-upon location. The questionnaire consisted of 2 sections. The trained interviewers administered part I and recorded the participants' answers about demographic information (e.g., living situation, family composition, education, measures of socioeconomic status). Part 2 of the survey focused on mental-health symptoms, substance and alcohol use, and nonsuicidal self-harm. To enhance the confidentiality of this section, each youth recorded his or her own answers. All surveys were placed in an envelope and sealed so that the interviewer did not see the confidential responses. Each youth received a Can $\$ 25$ gift certificate for their participation at each interview.

We used the Child and Adolescent Self-Harm in Europe definition modified to define nonsuicidal self-harm (Box I). Minor adjustments were made to the definition, including changing "initiated behaviour" to "self-injury" and "substance" to "medication." We also included in the survey additional questions asking the youth where they got the idea to self-harm and if they had sought help for this behaviour.

The Victoria Healthy Youth Survey also included sections of the Brief Child and Family Phone Interview. ${ }^{10}$ These questions were used to assess symptoms of anxiety (e.g., worries about doing better at things or about past behaviour, fears making a mistake); depressive mood (e.g., has no interest in his or her usual activities, feels hopeless, seems unhappy, sad or depressed); separation from parents (e.g., worries that bad things will happen to loved ones, becomes overly upset when leaving loved ones, complains of feeling sick before separating from loved ones); regulation of attention, impulsivity and activity (e.g., is distractable or has trouble sticking to an activity, is impulsive or acts without stopping to think, jumps from one activity to another, fidgets); cooperativeness (e.g., is defiant or talks back to adults, blames others for his or her mistakes, argues a lot with adults); and conduct (e.g., steals things at home, destroys things that belong to others, engages in vandalism, physically attacks people). Each symptom category included 6 related symptoms. Participants scored the frequency of each behaviour on a scale of $\mathrm{I}-3$ ( $\mathrm{I}=$ never, 2 = sometimes, $3=$ often). Construct and concur- rent validity have been demonstrated (Cronbach's $\alpha$ for individual domains varied from $0.62-0.8 \mathrm{I}$, indicating acceptable internal consistency). ${ }^{11}$

\section{Statistical analysis}

We used 2 methods to tabulate the data from the Brief Child and Family Phone Interview. First, we summed the number of symptoms reported in each category to form the total number of symptoms reported. Second, because the difference in the mean number of symptoms reported between those who reported nonsuicidal self-harm and those who did not was small for all categories except depressive mood symptoms, clinical meaningfulness was ambiguous. We therefore dichotomized the categories based on a count of the number of symptoms reported in each category ( $<5$ v. $\geq 5$ symptoms reported). We chose this high threshold for the number of reported symptoms per category to ensure that only those who reported a clinically important number of symptoms were represented. Missing data were minimal.

Demographic and help-seeking variables were analyzed by use of Student's $t$ test for continuous data and $\chi^{2}$ for binary or categorical data. We used multivariable analysis of variance with type III sum of squares to assess the differences in the total symptom scores for the Brief Child and Family Phone Interview between those who reported nonsuicidal self-harm and those who did not. We used logistic regression to predict the occurrence (v. nonoccurrence) of nonsuicidal self-harm, first on the basis of demographic variables and then with the addition of mental-health factors. The conduct category was excluded from this analysis because of the low number of participants who reported having 5 or more symptoms in this category. The standard errors for the parameter estimates and a tolerance test indicated that there was acceptable correlation among predictor variables. ${ }^{12}$ In the first logistic model, demographic variables that significantly distinguished between groups as well as age (because of its clinical importance) were entered into the model. In the second model, the dichotomized Brief Child and Family Phone Interview categories were added. We tested the logistic models for goodness of fit using the Hosmer-Lemeshow test $(p>0.05)$. An odds ratio greater than $\mathrm{I}$ indicates that nonsuicidal self-harm is more common in a given group than among those in the reference group.

\section{Box 1: Survey questions used to identify nonsuicidal self-harm*4,7}

Have you ever harmed yourself in a way that was deliberate but not intended as a means to take your life? Yes/ No (if No, this is the end of the survey)

If yes, which statement(s) best describe(s) this self-harm behaviour?

1. Self-injury such as self-cutting, self-scratching, self-hitting etc? Yes / No

2. Ingesting a medication in excess of the prescribed or generally recognized therapeutic dose? Yes / No

3. Ingesting a recreational or illicit drug or alcohol as a means to harm yourself? Yes / No

4. Ingesting a non-ingestible substance or object? Yes / No

5. Other? please specify:

Yes / No

*Definition of nonsuicidal self-harm was modified from the Child and Adolescent Self-Harm in Europe (CASE) definition of deliberate self-harm. 


\section{Results}

In the first wave of the survey in 2003, which did not include questions about nonsuicidal self-harm, a random sample of 9500 telephone listings identified 1036 households with an eligible youth (aged I2-I8) (Figure I). Of these, $\mathrm{I} 85$ (I7.9\%) parents or guardians and $\mathrm{I} 87$ (I8.I\%) youths refused to participate. Demographic data were not available on those who refused. Complete data were available for the 664 youth who participated in the first wave of data collection in 2003 and from the $580(87.3 \%)$ youth who participated in a second wave in 2005 (self-harm questions were added to wave 2 of the survey) (Figure I). In total, $568(97.9 \%)$ youth completed the self-injury selfreport section of the survey. Males were significantly less

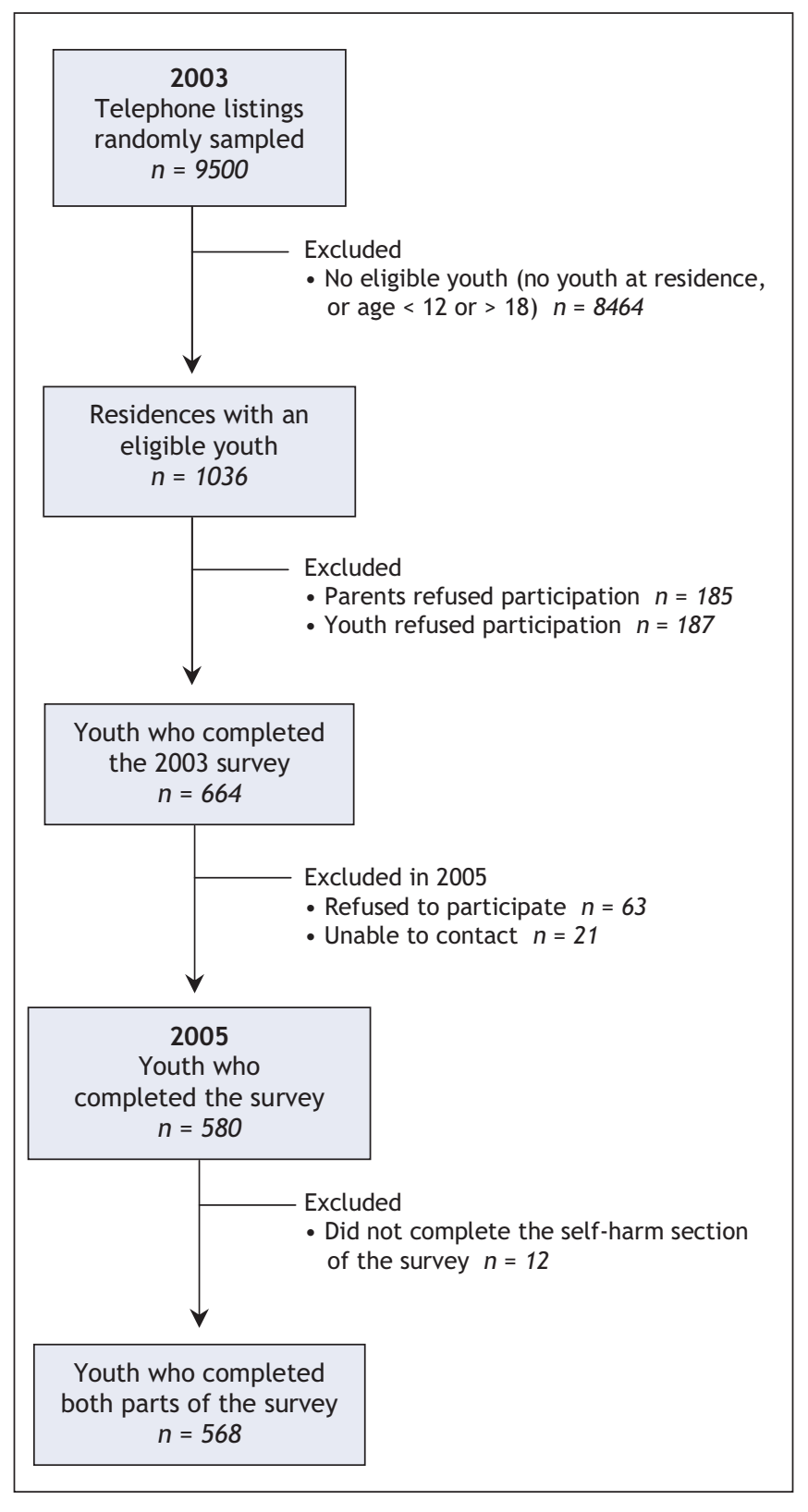

Figure 1: Flow of participants in the study. likely than females to complete the self-harm section of the survey $(p=0.013)$.

Of the 568 youth who completed the nonsuicidal self-harm section, 96 (I6.9\%) reported that they had ever harmed themselves deliberately. The act of nonsuicidal self-harm occurred I time for 27 (29.0\%) youths, $2-3$ times for 3I (33.3\%) youths and more than 3 times for 35 (37.6\%) youths. The most frequently reported forms of nonsuicidal self-harm (descending order) were: self-injury such as cutting, scratching and selfhitting $(79 / 95,83.2 \%)$, ingesting a medication in excess of the prescribed or generally recognized dosage $(28 / 89,31.5 \%)$, ingesting a recreational or illicit drug or alcohol as a means to harm self ( $15 / 86,16.9 \%)$ and other nonspecified forms of selfinjury $(8 / 85,9.4 \%)$. The mean number of nonsuicidal selfharm incidents was $\mathrm{I} .4$ (standard deviation [SD] 0.7 , min-max I-4). The mean age of onset was 15.2 years (SD 2.2, min-max IO-20), and the mean duration for those who had stopped was I.8 years (SD I.6, min-max o-4.5).

The majority of those who self-harmed reported that the idea to self-harm had been their own $(69 / 93,73.4 \%)$. Few youths reported that they had gotten the idea from a friend $(27 / 93,29.0 \%)$, television or movies (I4/93, 15.1\%) or a family member $(2 / 93,2.2 \%)$, or that they had read about it (II/93, II.8\%). For the question "where did you get the idea to self-harm," the mean number of sources reported was I.4 (SD o.9).

Of the youth who reported nonsuicidal self-harm, $56 \%$ (5I/9I) indicated that they had sought help or support. These youths reported seeking help from friends $(28 / 50,56.0 \%)$, a psychiatrist or psychologist (27/50, 54.0\%), family (24/50, $48.0 \%)$, other mental health professionals $(\mathrm{I} 6 / 50,32.0 \%$ ), family doctor ( $15 / 50,30.0 \%$ ), other non-specified sources of help (I4/50, 28.0\%) and help lines (9/50, $18.0 \%)$. The mean number of sources of support reported was 2.9 (SD I.6). Help-seeking was positively associated with the frequency of nonsuicidal self-harm $(p=0.04)$.

There were significant differences between those who reported nonsuicidal self-harm and those who did not for sex and socioeconomic status (Table I). Females were more likely than males to self-harm, and youth who reported that their family had problems affording the basic necessities were more likely to report nonsuicidal self-harm than those who did not report self-harm. There was no difference in the proportion of youths enrolled in school between the self-harm and no self-harm groups. Of the Iog youth who were not currently enrolled in school, $37(33.9 \%)$ had graduated from high school, 39 (35.8\%) had dropped out for personal reasons (including medical issues, suspension or expulsion and a need for money) and 33 (30.3\%) had dropped out for other nonidentified reasons.

The mean number of symptoms reported for each of the 6 categories was significantly different between those who reported nonsuicidal self-harm and those who did not $(p<0.00 I)$. There were statistically significant differences between the self-harm and no self-harm groups on every Brief Child and Family Phone Interview category, with the selfharm group reporting a higher mean number of symptoms in each category (Table 2, Table 3). 
When we analyzed the data using the first model, in which demographic variables that significantly distinguished between groups, as well as age (because of its clinical importance), sex was the only significant predictor of nonsuicidal self-harm, with females being at a higher risk than males. In the second model, the dichotomized Brief Child and Family Phone Interview categories were added and were found to have significant predictive value for nonsuicidal self-harm. In the latter model, sex remained a significant predictor of nonsuicidal self-harm. Depressive mood symptoms and prob-

Table 1: Self-reported characteristics of 568 participants in the Victoria Healthy Youth survey, a population-based survey of youth

Self-reported nonsuicidal self-harm, no. $(\%)$ of participants

\begin{tabular}{|c|c|c|c|c|}
\hline \multirow[b]{2}{*}{ Characteristic } & \multirow[b]{2}{*}{ No. of participants } & \multirow{2}{*}{$\begin{array}{c}\text { Yes } \\
n=96\end{array}$} & & \multirow[b]{2}{*}{$p$ value } \\
\hline & & & $\begin{array}{c}\text { No } \\
n=472\end{array}$ & \\
\hline Male & 263 & $22(22.9)$ & $241 \quad(51.1)$ & 0.001 \\
\hline Female & 305 & 74 (77.1) & $231 \quad(48.9)$ & \\
\hline \multicolumn{5}{|l|}{ Age, yr } \\
\hline $16-17$ & 173 & $25(14.5)$ & $148 \quad(85.5)$ & \\
\hline $18-19$ & 186 & $40 \quad(21.5)$ & $146(78.5)$ & \\
\hline $20-21$ & 72 & $10(13.9)$ & $62(86.1)$ & \\
\hline \multicolumn{5}{|l|}{ Current living situation } \\
\hline With 1 parent & 121 & $17(19.3)$ & $104(22.4)$ & 0.32 \\
\hline Yes & 458 & 74 (77.1) & 384 (81.5) & 0.31 \\
\hline No & 109 & $22(22.9)$ & $87 \quad(18.5)$ & \\
\hline \multicolumn{5}{|l|}{ Currently employed } \\
\hline Full time & 104 & $16(16.7)$ & $88 \quad(18.6)$ & 0.49 \\
\hline Part time & 223 & $34(35.4)$ & $189(40.0)$ & \\
\hline Not employed & 241 & $46 \quad(47.9)$ & $195(41.3)$ & \\
\hline \multicolumn{5}{|c|}{$\begin{array}{l}\text { Family money problems for } \\
\text { basic necessities }\end{array}$} \\
\hline Never & 466 & 71 (75.5) & $395 \quad(84.8)$ & 0.029 \\
\hline
\end{tabular}

Table 2: Number of symptoms reported by 568 participants for each Brief Child and Family Phone Interview category in the Victoria Healthy Youth Survey, a population-based survey of youth

\begin{tabular}{llll}
\hline & \multicolumn{2}{c}{$\begin{array}{c}\text { Nonsuicidal self-harm, } \\
\text { mean no. (SD) of symptoms reported }\end{array}$} \\
\cline { 2 - 4 } Category* & $\begin{array}{c}\text { Yes } \\
n=96\end{array}$ & $n=472$ & $2.8(1.7)$ \\
\hline Separation from parents & $3.5(1.7)$ & $4.7(1.4)$ & 0.001 \\
\hline Anxiety symptoms & $5.1(1.1)$ & $2.5(2.0)$ & 0.002 \\
\hline Depressive mood symptoms & $4.2(1.7)$ & $3.5(1.7)$ & 0.001 \\
\hline Cooperativeness & $4.4(1.4)$ & $3.7(1.7)$ & 0.001 \\
\hline Regulation of attention, impulsivity and activity & $4.4(1.6)$ & $0.3(0.7)$ \\
\hline Conduct & $0.7(1.2)$ & 0.001 & 0.001 \\
\hline
\end{tabular}

Note: $\mathrm{SD}=$ standard deviation

*Each category contains 6 related symptoms. 
Table 3: Participants who reported having 5 or more symptoms or less than 5 symptoms in each category in the Victoria Healthy Youth Survey, a population-based survey of youth

\begin{tabular}{|c|c|c|}
\hline \multirow[b]{2}{*}{ Symptom category* } & \multicolumn{2}{|c|}{ Nonsuicidal self-harm, no. (\%) of participants } \\
\hline & $\begin{array}{c}\text { Yes } \\
n=96\end{array}$ & $\begin{array}{c}\text { No } \\
n=472\end{array}$ \\
\hline \multicolumn{3}{|c|}{ Separation from parents, no. of symptoms reported } \\
\hline$\geq 5$ & $36(37.5)$ & $97(20.6)$ \\
\hline$<5$ & $60(62.5)$ & $375(79.4)$ \\
\hline$\geq 5$ & $75(78.1)$ & $291(61.7)$ \\
\hline$<5$ & $21(21.9)$ & $181(38.3)$ \\
\hline \multicolumn{3}{|c|}{$\begin{array}{l}\text { Depressive mood symptoms, } \\
\text { no. of symptoms reported }\end{array}$} \\
\hline$\geq 5$ & 45 (46.9) & $97(20.6)$ \\
\hline$<5$ & $47(49.0)$ & $318(67.4)$ \\
\hline \multicolumn{3}{|c|}{$\begin{array}{l}\text { Regulation of attention, impulsivity and activity, } \\
\text { no. of symptoms reported }\end{array}$} \\
\hline$\geq 5$ & $60(62.5)$ & $179(37.9)$ \\
\hline$<5$ & $36(37.5)$ & $293(62.1)$ \\
\hline \multicolumn{3}{|c|}{ Conduct, no. of symptoms reported } \\
\hline$\geq 5$ & $1(1.0)$ & $1(0.2)$ \\
\hline$<5$ & $95(99.0)$ & $471(99.8)$ \\
\hline
\end{tabular}

*Each symptom category contains 6 related symptoms.

lems with regulation of attention, impulsivity and activity were also significant predictors. Specifically, youth who reported 5 or more symptoms in these categories were more likely to self-harm than youth who reported fewer than 5 symptoms (Table 4).

\section{Interpretation}

We found that the lifetime prevalence of nonsuicidal selfharm among youth in our sample (including about $20 \%$ not enrolled in school) was $16.9 \%$ and that the mean age of onset was 15 years. Of those who reported nonsuicidal self-harm, $77 \%$ were female and $40 \%$ reported harming themselves repeatedly ( $>3$ times). Almost $75 \%$ of youth reported that the idea for nonsuicidal self-harm had come from themselves, and fewer youth indicated that the idea had come from friends ( $29 \%$ ), family $(2 \%)$ or the media (television or movies, $15 \%$; read about it, $12 \%$ ). Just over half of the youth who reported nonsuicidal self-harm also reported that they had sought help for this behaviour, turning primarily to friends or, less often, to a psychiatrist or psychologist. Youth who reported more frequent nonsuicidal self-harm were more likely to report seeking help for this behaviour. Youth who indicated nonsuicidal self-harm were more likely than those who did not report nonsuicidal self-harm to also report having de- pressive mood symptoms and problems with regulation of attention, impulsivity and activity.

The prevalence of nonsuicidal self-harm in our study was similar to the rates reported in 2 Canadian studies of nonsuicidal self-harm in school-based youth, a recent US survey of college students, and British and Australian surveys of "deliberate" self-harm. ${ }^{4}$ Among students at urban schools in large metropolitan areas of Canada, the lifetime prevalence of selfharm was reported to be $13.9 \%$ in Eastern Canada and $15 \%$ in Western Canada. ${ }^{5,8}$ An Internet-based survey of students at 2 universities in the northeastern United States found a lifetime prevalence of self-injury (defined as having engaged in at least I of I6 identified methods of self-injury) of $17 \%{ }^{6}$ De Leo and Heller reported a lifetime prevalence of "deliberate" selfharm of $12.4 \%$ in a school-based survey of Australian youth, ${ }^{4}$ and Hawton and colleagues found a similar rate ( $13.2 \%)$ in a cross-sectional, self-report survey of students aged I5-I6 years in England. ${ }^{7}$ In contrast, surveys of Norwegian and Hungarian youth have reported a lifetime prevalence of selfharm of $8.1 \%$ and I0.I\% respectively. ${ }^{13,14}$

We found that the most common type of reported nonsuicidal self-harm was self-injury (e.g., cutting, scratching, selfhitting). The reported frequencies of the types of self-harm are difficult to compare with previous studies because of the lack of consistency in definition and surveying methods. In 
Table 4: Logistic regression of self-harming behaviour among 560 youth* who responded to the nonsuicidal self-harm questions on the Victoria Healthy Youth Survey, a population-based survey of youth

Odds ratio ( $95 \%$ confidence interval)

\begin{tabular}{|c|c|c|c|c|}
\hline \multirow{3}{*}{$\begin{array}{l}\text { Variable } \neq \\
\text { Sex (female v. male) }\end{array}$} & \multirow{2}{*}{\multicolumn{2}{|c|}{ Model $1 \dagger$}} & \multirow{2}{*}{\multicolumn{2}{|c|}{ Model $2 \dagger$}} \\
\hline & & & & \\
\hline & $3.33 \S$ & $(1.99-5.56)$ & $3.72 \S$ & $(2.16-6.38)$ \\
\hline Family money problems for basic necessities (yes v. no) & 0.63 & $(0.36-1.08)$ & 0.93 & $(0.52-1.69)$ \\
\hline Age (per yr) & 0.99 & $(0.99-1.01)$ & 0.99 & $(0.99-1.01)$ \\
\hline \multicolumn{5}{|l|}{ Symptom category $\dagger$ ( $\geq 5$ v. $<5$ symptoms reported) } \\
\hline Separation from parents & & & 1.25 & $(0.73-2.15)$ \\
\hline Anxiety symptoms & & & 1.48 & $(0.84-2.63)$ \\
\hline Depressive mood symptoms & & & 2.189 & $(1.28-3.71)$ \\
\hline Cooperativeness & & & 1.35 & $(0.80-2.28)$ \\
\hline Regulation of attention, impulsivity and activity & & & $2.24^{* *}$ & $(1.33-3.76)$ \\
\hline
\end{tabular}

*Eight cases were excluded because of missing data.

tGoodness of fit was demonstrated by no significant departure of model predictions from the observed data (Hosmer-Lemeshow test, $p>0.05$ ).

†The conduct symptom category was excluded from this analysis because of the low number of participants who reported having $\geq 5$ symptoms in this category. $\S p<0.001$.

१p $=0.004$

${ }^{* *} p=0.002$

our study, the rate of self-injury was higher compared with rates reported in the United Kingdom (64.6\%) and Australia (6I.4\%), which may be because of differences in sampling and definitions.

The strong association between depressive mood symptoms and nonsuicidal self-harm among youth with a history of this behaviour is consistent with research involving youth in both the community and in hospital whose affect regulation to relieve feelings of depression or to reduce tension was a primary function of the behaviour. ${ }^{7,8,13,15,16}$ Impulsive behaviour has been noted to be strongly associated with nonsuicidal self-harm. ${ }^{4,7}$ We found that slightly more than half of youth who reported ever self-harming had sought help for this behaviour. This rate is higher than that reported by Evans and colleagues ${ }^{17}$ for "deliberate" self-harm among youth in the United Kingdom but is similar to the rate of nonsuicidal self-injury among college students. ${ }^{6}$ The rate in our study is also similar to that observed in a Canadian population-based study of youth investigating service use among adolescents with major depression and suicidality. ${ }^{18}$

This study has several limitations. The data are based on a regional sample of youth and therefore not necessarily generalizable across Canada. Data were not available for those who refused to participate in the initial 2003 survey; therefore, we cannot compare the characteristics of those who participated and those who refused. It is possible that individuals who refused to participate had higher levels of symptomatology and that our results may underestimate the prevalence of nonsuicidal self-harm and overestimate helpseeking behaviour.

The data used in our study were cross-sectional, retrospective and self-reported. Thus, we cannot determine whether our findings of a strong association between nonsuicidal selfharm, depressive mood symptoms and problems of regula- tion of attention, impulsivity and activity are causal, consequential or coincidental. The prevalence of nonsuicidal selfharm reported here may be an underestimate, given that for some youth the onset of nonsuicidal self-harm may have not yet occurred and that others may have been unwilling to report such behaviour. Future research should use a national sample and a longitudinal approach with information gathered from multiple respondents.

Whether a youth with nonsuicidal self-harm seeks help may depend on several factors, including attitudes toward nonsuicidal self-harm and seeking mental health services, availability and accessibility of services, attention paid to screening for this behaviour when a professional assessment or encounter occurs, and whether youth are willing to disclose and to whom they might disclose. Epidemiologic studies, such as that by Lin and colleagues, ${ }^{19}$ that have examined the use of mental-health services in Ontario may provide more detailed information about help-seeking patterns in such youth. These studies may further inform health policy and practice for nonsuicidal self-harm.

In conclusion, the results of our study suggest that nonsuicidal self-harm in youth is common. If nonsuicidal self-harm is identified, the type and frequency should be further evaluated, and youth should be assessed for associated risk factors. Identification of mental health and/or behavioural difficulties and information about the youth's reasons for nonsuicidal self-harm can help health care providers understand the behaviour in context and determine a suitable approach. Health care professionals should be vigilant in assessing for nonsuicidal self-harm in youth who present with mental health issues (Box I).

This article has been peer reviewed.

Competing interests: None declared. 
Contributors: All of the authors contributed substantially to the study conception and design and to the interpretation of the data. Mary K. Nixon and Mikael Jansson were directly involved in the acquisition of the data. All of the authors were jointly involved in the analysis of the data. Mary K. Nixon and Paula Cloutier drafted the manuscript and, with Mikael Jansson, revised it critically for important intellectual content. All of the authors gave approval of the final version submitted for publication.

Acknowledgements: We thank the researchers and research assistants affiliated with the Centre for Youth and Society at the University of Victoria and many of the community partners who assisted in developing the Victoria Healthy Youth Survey. We also thank the research assistants who helped in the data collection and the youth who completed the survey. Dr. Nixon thanks Drs. Leadbeater and Banister for their coverage of safety issues when she was not available.

This research was funded by a Community Alliance for Health Research grant from the Canadian Institutes for Health Research (grant \#CAR-43275).

\section{REFERENCES}

I. Favazza AR, Conterio K. Female habitual self-mutilators. Acta Psychiatr Scand I989;79:283-9.

2. Herpertz S. Self-injurious behaviour: Psychopathological and nosological characteristics in subtypes of self-injurers. Acta Psychiatr Scand i995;9I:57-68.

3. Klonsky ED. The functions of deliberate self-injury: A review of the evidence. Clin Psychol Rev 2007;27:226-39.

4. De Leo D, Heller ST. Who are the kids who self-harm? An Australian self-report school survey. Med J Aust 2004;18I:I40-4.

5. Laye-Gindhu A, Schonert-Reichl K. Nonsuicidal self-harm among community adolescents: understanding the "whats" and "whys" of self-harm. J Youth Adolesc 2005;34:447-57.

6. Whitlock J, Eckenrode J, Silverman D. Self-injurious behaviors in a college population. Pediatrics 2006;117:1939-48.

7. Hawton K, Rodham K, Evans E, et al. Deliberate self harm in adolescents: self report survey in schools in England. BMJ 2002;325:1207-II.
8. Ross $\mathrm{S}$, Heath N. A study of the frequency of self-mutilation in a community sample of adolescents. J Youth Adolesc 2002;31:67-77.

9. Jansson M, Mitic W, Russell T, et al. A youth population health survey. In: Leadbeater BJ, Banister T, Riecken J, et al., editors. Ethical issues in community-based research with children and youth. Toronto: University of Toronto Press; 2006. p. 59-62.

Io. Cunningham C, Pettingill $\mathrm{P}$, Boyle $\mathrm{M}$. The brief child and family phone interview. Hamilton (ON): McMaster University; 200I.

II. Cunningham C, Pettingill P, Boyle M. Evaluating the BCFPI-3: adolescent interview. In: The brief child and family phone interview (BCFPI-3). A computerized in take and outcome assessment tool: interviewers manual. Hamilton (ON): McMaster University; 2006. p. 94-9.

I2. Tabachnick B, Fidell L. Using multivariate statistics. New York: HarperCollins I996.

I3. Groholt B, Ekeberg O, Wichstrom L, et al. Young suicide attempters: A comparison between a clinical and an epidemiological sample. J Am Acad Child Adolesc Psychiatry 2000;39:868-75.

I4. Fekete S, Voros V, Osvath P. Suicidal behaviour and psychopathology in adolescents: results of a self-report survey among 15 and 16 year old adolescent people in hungary. Eur Neuropsychopharmacol 2004; I4 $_{4} \mathrm{~S}_{3} 65$.

15. Muehlenkamp JJ, Gutierrez PM. An investigation of differences between self-injurious behavior and suicide attempts in a sample of adolescents. Suicide Life Threat Behav 2004;34:12-23.

I6. Nixon MK, Cloutier PF, Aggarwal S. Affect regulation and addictive aspects of repetitive self-injury in hospitalized adolescents. J Am Acad Child Adolesc Psychiatry 2002;4I:I333-4I.

17. Evans E, Hawton K, Rodham K. In what ways are adolescents who engage in selfharm or experience thoughts of self-harm different in terms of help-seeking, communication and coping strategies? J Adolesc 2005;28:573-87.

I8. Cheung AH, Dewa CS. Mental heath service use among adolescents and young adults with major depressive disorder and suicidality. Can J Psychiatry 2007:52:228-32.

19. Lin E, Goering P, Offord RD, et al. The use of mental health services in Ontario: epidemiologic findings. Can J Psychiatry 1996 6;41:572-7.

Correspondence to: Dr. Mary K. Nixon, Centre for Youth and Society, University of Victoria, PO Box I70o Stn. CSC, Victoria BC V8W 2Y2; fax 250 72I-8929; mary.nixon@viha.ca

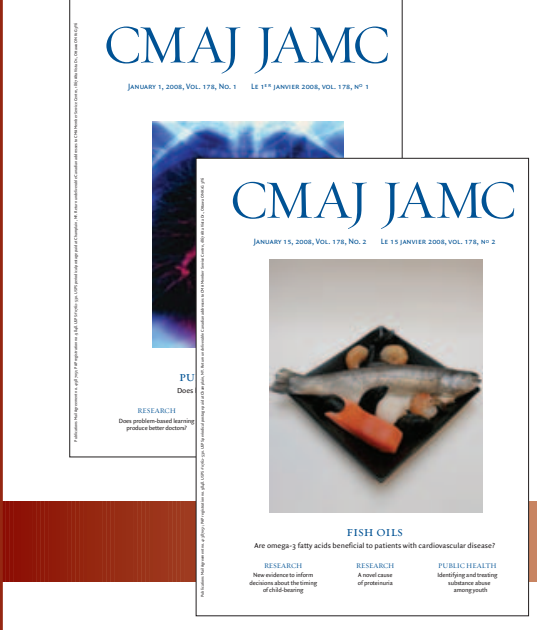

\section{Online manuscript submission and peer review available for $C M A J$}

